\title{
Prospects for 3D Imaging of Dopant Atoms in Ceramic Materials
}

\author{
S.D. Findlay, $*$ N. Shibata,*** S. Azuma* and Y. Ikuhara********* \\ * Institute of Engineering Innovation, School of Engineering, The University of Tokyo, Tokyo, 113- \\ 8656, Japan \\ ** PRESTO, Japan Science and Technology Agency, 4-1-8 Honcho Kawaguchi, Saitama, 332-0012, \\ Japan \\ *** Nanostructures Research Laboratory, Japan Fine Ceramics Center, 2-4-1 Mutsuno, Atsuta, \\ Nagoya, 456-8587, Japan \\ **** WPI Advanced Institute for Materials Research, Tohoku University, 2-1-1, Katahira, Aoba, \\ Sendai, 980-8577, Japan
}

High angle annular dark field (HAADF) imaging in scanning transmission electron microscopy (STEM) is well suited to identifying heavy elements in lighter surrounds [1,2]. This has been used recently to explore the detailed configuration of impurity atoms segregated at grain boundaries in ceramic materials [3], where such dopant addition can significantly change the physical properties of the material, particularly its resistance to creep deformation [4]. At best, a single image only gives a clear indication of the projected structure along one direction. However the complexity of interface structure makes it desirable to seek further information, such as the dopant distribution. Image quantification may provide one approach [5-7], and depth sectioning in aberration corrected machines may provide another [8]. However, the most direct approach is to observe the specimen from multiple directions, provided the images can be interpreted equally well. A spherical aberration corrector allows the formation of an atomically fine probe on the crystal surface, but, depending on the specimen orientation, it becomes important to describe in detail how the probe spreads through the sample in order to best interpret the experimental images. We will explore the effects of sample orientation, defocus selection, and probe spreading on atomic resolution HAADF STEM imaging using rare earth segregation in an alumina $\left(\alpha-\mathrm{Al}_{2} \mathrm{O}_{3}\right)$ grain boundary as a test case.

We explore imaging of the dopant distribution in the interface plane via imaging from three orthogonal directions with a view to determining as much three-dimensional structure information as possible. Fig. 1 shows the projected structure model and corresponding simulated HAADF images for a $\mathrm{Y}$-doped $\alpha-\mathrm{Al}_{2} \mathrm{O}_{3}$ grain boundary between two single crystals in the $\Sigma 13$ orientation relationship. Figs. $1 \mathrm{~A}$ and $\mathrm{B}$ have the crystals viewed along the $<1 \overline{2} 10>$ direction while Figs. 1C and $\mathrm{D}$ have it viewed along the $<\overline{2} 021>$ direction. In both cases the $<50 \overline{5} 4>$ direction is vertical in the images. As the matrix is fairly light and the $<50 \overline{5} 4>$ direction is not a strong channelling direction, it may be possible to image the interface from the $<50 \overline{5} 4>$ direction as well. Fig. $2 \mathrm{~A}$ shows the intensity profile, projected along the $<2021>$ direction, of the electron probe wavefunction travelling along the $<50 \overline{5} 4>$ direction through the two crystals of total thickness $325 \AA$. Fig. $2 \mathrm{~B}$ shows the corresponding profile in vacuum. The differences between the profiles are small, suggesting that the crystal does not cause much perturbation of the intensity distribution and that the probe retains its atomic sized beam waist at the interface. Fig. 2C shows the simulated HAADF STEM image of the model $\mathrm{Y}$-doped $\alpha-\mathrm{Al}_{2} \mathrm{O}_{3}$ grain boundary from this third orthogonal orientation, and despite the thickness of the sample the individual $\mathrm{Y}$ atoms are clearly visible: in this specimen it should be possible to image the buried interface in plan-view. 


\section{References}

[1] S.J. Pennycook and D.E. Jesson, Ultramicroscopy 37 (1991) 14.

[2] P.M. Voyles et al., Nature 416 (2002) 826.

[3] J.P. Buban et al., Science 311 (2006) 212.

[4] H. Yoshida, Y. Ikuhara and T. Sakuma, Acta Materialia 50 (2002) 2955.

[5] J.M. LeBeau, S.D. Findlay, L.J. Allen and S. Stemmer, Phys. Rev. Lett. 100 (2008) 206101.

[6] P.D. Robb and A.J. Craven, Ultramicroscopy 109 (2008) 61.

[7] S.I. Molina et al., Ultramicroscopy 109 (2009) 172.

[8] K. van Benthem et al., Appl. Phys. Lett. 87 (2005) 034104.

[9] S.D. Findlay was supported as a Japan Society for Promotion of Science (JSPS) fellow. N.

Shibata acknowledges support by Industrial Technology Research Grant program in 2007 from New

Energy and Industrial Technology Development Organization (NEDO) of Japan.
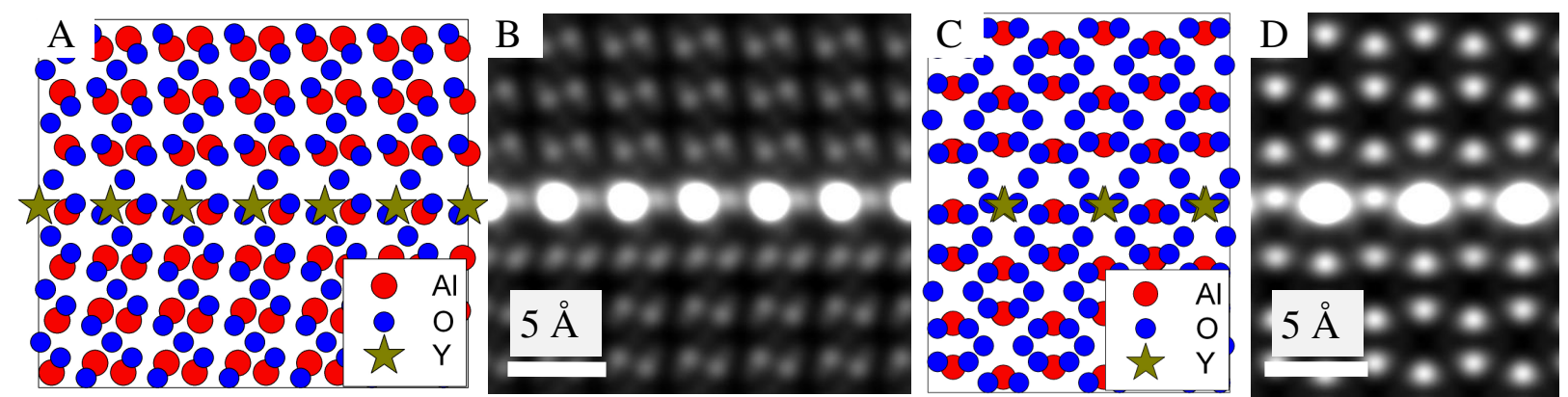

Fig. 1. A. Structure model and B. simulated HAADF STEM image of a $143 \AA$ thick Y-doped $\alpha$ $\mathrm{Al}_{2} \mathrm{O}_{3} \Sigma 13$ grain boundary viewed along the $<1210>$ zone axis orientation. C. Structure model and D. simulated HAADF STEM image of a $140 \AA$ thick Y-doped $\alpha-\mathrm{Al}_{2} \mathrm{O}_{3} \Sigma 13$ grain boundary viewed along the $<2021>$ zone axis orientation. Simulations assume a $200 \mathrm{keV}$, aberration-free probe with probe-forming aperture angle $27.4 \mathrm{mrad}$ and an ADF collector range of 81-228 mrad. The intensity range has been limited to make the crystal lattice visible, saturating the signal at the Y columns.
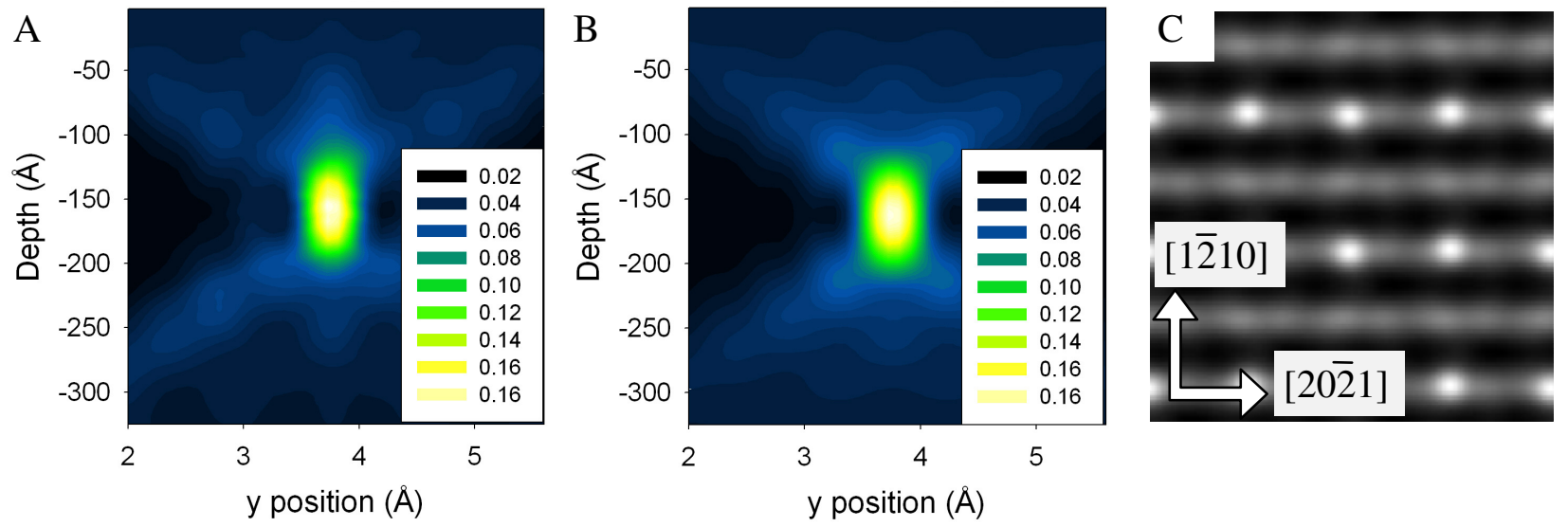

Fig. 2. The simulated intensity profiles of the electron beam, projected along the $<\overline{2} 021>$ direction, in A. focused on the interface plane between the crystals and in B. complete vacuum. C. HAADF image of the Y-doped grain boundary in plan-view, i.e. viewed along the $<50 \overline{5} 4>$ direction. The thickness of the sample is assumed to be $325 \AA$, and the defocus is set to the mid-plane (i.e. the interface plane) of the specimen. Other parameters are as given in the caption to Fig. 1. 